\title{
A Frequency-Tunable SRR-Adopted Two-Pole Waveguide Filter Operating below the Cutoff Frequency
}

\author{
Wook-Ki Park, ${ }^{1}$ Hyun-Yong Lim, ${ }^{1}$ Dong-Woo Kim, ${ }^{2}$ and Soon-soo $\mathrm{Oh}^{2}$ \\ ${ }^{1}$ Future Industry Technology Center, IBITP, 12 Gaetbeol-ro, Yeonsu-gu, Incheon 21999, Republic of Korea \\ ${ }^{2}$ Electronic Engineering, Chosun University, 309 Pilmundae-ro, Dong-gu, Gwangju 61452, Republic of Korea \\ Correspondence should be addressed to Soon-soo Oh; ssoh@chosun.ac.kr
}

Received 6 March 2017; Accepted 23 March 2017; Published 6 April 2017

Academic Editor: Luciano Tarricone

Copyright (c) 2017 Wook-Ki Park et al. This is an open access article distributed under the Creative Commons Attribution License, which permits unrestricted use, distribution, and reproduction in any medium, provided the original work is properly cited.

\begin{abstract}
A frequency-tunable filter operating below the cutoff frequency of a metallic hollow waveguide is proposed, which has two-pole bandpass characteristics and incorporates a split-ring resonator (SRR). The tuning procedure is achieved by inserting two metal screws. The screws adjust the magnetic- field passing through the center of the SRR. The effect of SRR orientation was investigated and the optimum orientation proposed. Our results indicated an operating frequency range of 2.0 to $2.35 \mathrm{GHz}$, which is lower than the cutoff frequency of the waveguide of $4.9 \mathrm{GHz}$ by more than $2.55 \mathrm{GHz}$. The proposed filter could be utilized for communication, such as software-defined radio (SDR) systems.
\end{abstract}

\section{Introduction}

Recently, frequency-tunable devices have been researched intensively for microwave applications, in an effort to meet the demands of numerous wireless services [1-3]. As well as frequency tunability, a microwave filter must be lightweight and compact [4]. Waveguide filters are utilized for highpower applications; however, their operating frequency is above the cutoff frequency, which is governed by the width of the waveguide. The usual method for waveguide miniaturization is to reduce the waveguide width and then implement an evanescent-mode filter, inserting a capacitive obstacle such as a screw and a dielectric sheet [5,6]. A more recent technique for the miniaturization of waveguide filters is based on the insertion of a split-ring resonator (SRR) array into the waveguide [7-9]. An improved technique using a metal or ferrite tuning screw for an SRR-loaded waveguide was proposed in [10].

The SRR-loaded waveguide filter technique differs from that of the evanescent-mode filter. Below the cutoff frequency, the equivalent circuit of a SRR-loaded waveguide filter is mainly configured as a series capacitor and a parallel inductor, whereas the equivalent circuit of an evanescentmode filter is configured as a parallel inductor and a parallel capacitor. This difference is explained in detail in [10].
In this paper, a frequency-tunable waveguide filter operating below the cutoff frequency is proposed, with two-pole bandpass characteristics. The proposed filter is an expanded version of the one-pole bandpass filter described in [10]. This miniaturized waveguide adopts two SRRs with optimized orientations. The simulation and experimental results show the frequency tunability of the two poles.

\section{Design of Two-Pole Filter}

The proposed waveguide two-pole filter is illustrated in Figure 1(a), which gives a perspective view; Figure 1(b) provides a side view. The tunable frequency was set from $2.0 \mathrm{GHz}$ to $2.35 \mathrm{GHz}$, as this device is a modification of the filter described in [10], which was originally set for ease of manufacturing and testing. Two SRRs were utilized to implement two-pole characteristics. The substrate containing two rings was placed along the central line of the hollow square waveguide, as shown in Figure 1(a). The spacing between the two SRRs was $s=38 \mathrm{~mm}$. To tune the operating frequency of the bandpass filter, two metallic screws were inserted vertically through the side of the hollow square waveguide: $n$ in Figures 1(a) and 1(b). The rings were printed on a Rogers RT/duroid ${ }^{\circledR}-5880$ substrate of thickness 


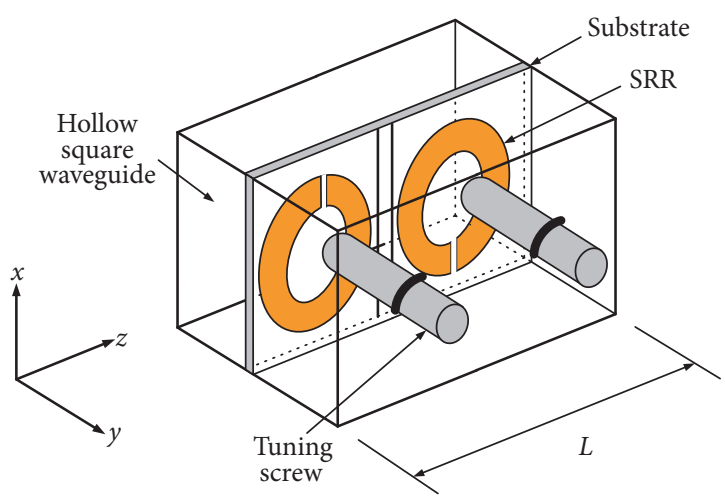

(a)
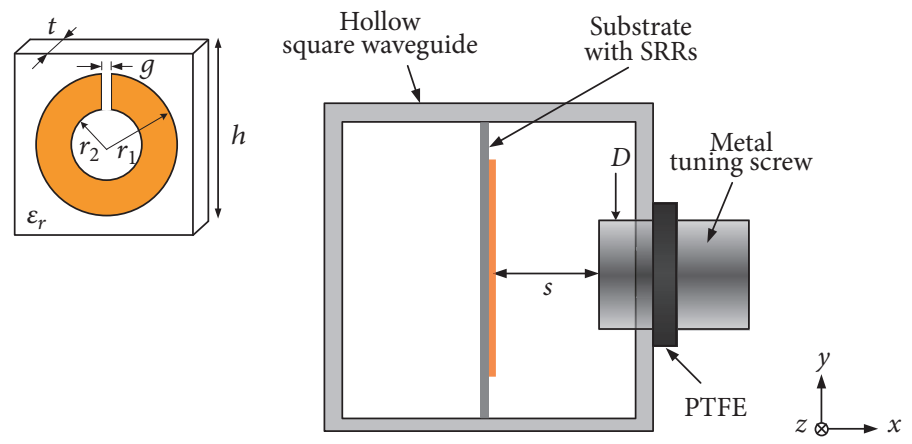

(b)

FIGURE 1: Substrate loaded with an SRR and tuning screw: (a) prospective and SRR, (b) side view.

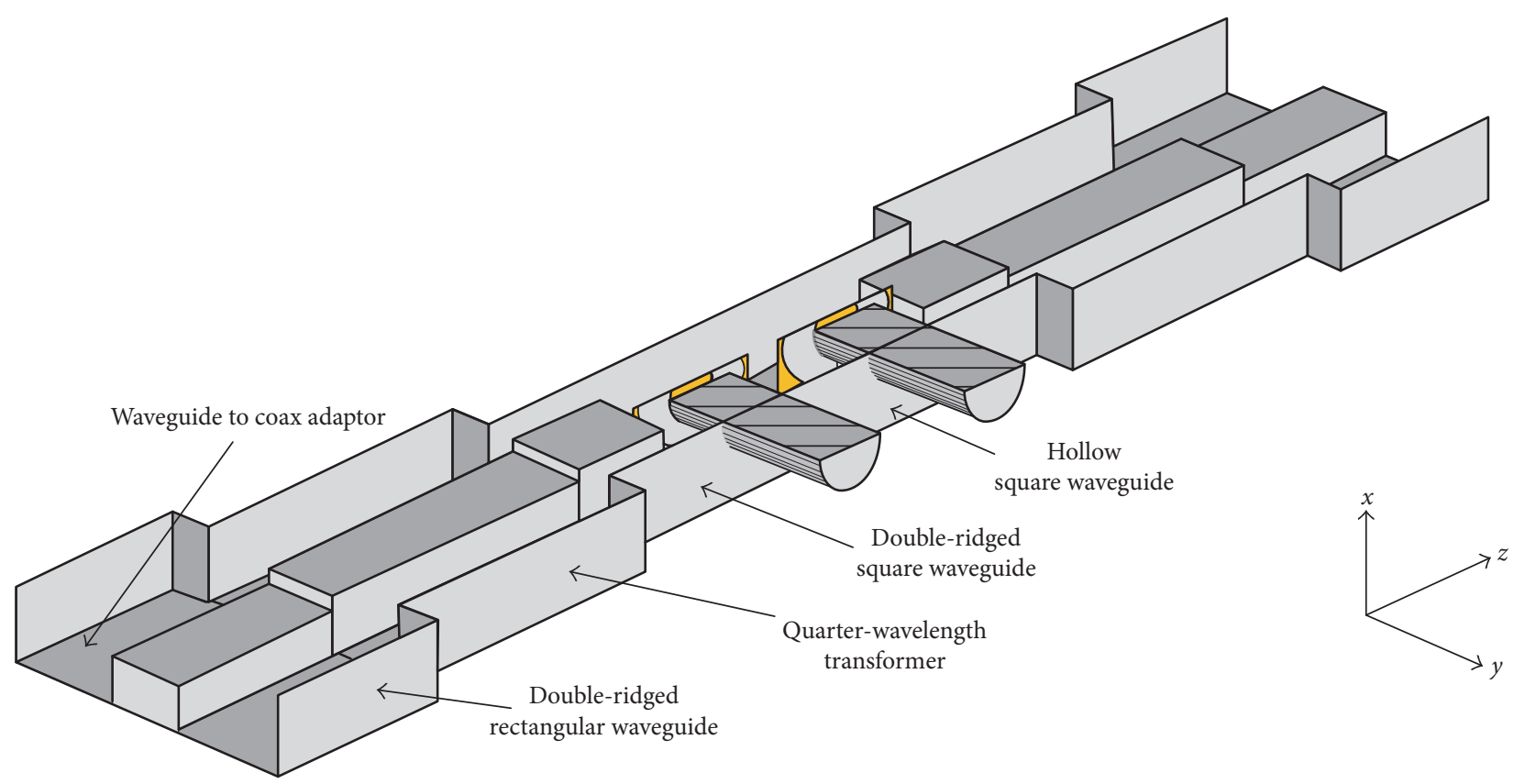

FIGURE 2: Half-cut structure of the proposed two-pole waveguide.

$t=0.508 \mathrm{~mm}$, dielectric constant $\varepsilon_{r}=2.2$, and tangent loss $\tan \delta=0.009$. The ring had an outer radius $r_{1}=14.0 \mathrm{~mm}$, an inner radius $r_{2}=6 \mathrm{~mm}$, and a gap $g=1.6 \mathrm{~mm}$.

The length of the substrate was $L=68 \mathrm{~mm}$, equal to the length of the hollow square waveguide. The height of the hollow square waveguide was $30.61 \mathrm{~mm}$, equal to the height of the substrate $h$. The width of the hollow square waveguide was also $30.61 \mathrm{~mm}$, corresponding to a cutoff frequency of $4.9 \mathrm{GHz}$. Hence, the design frequency was much lower than the cutoff frequency, by more than $2.55 \mathrm{GHz}$. This aperture was miniaturized by about $70 \%$ compared with the apertures of standard waveguide filters, with dimensions of $47.55 \mathrm{~mm}$ $\times 22.15 \mathrm{~mm}$. The diameter of the metallic tuning screw was $D=8.0 \mathrm{~mm}$, as shown in Figure 1(b). This tuning screw disturbs the magnetic field passing through the center of the ring [11].
To feed the wave from the waveguide-to-coax adaptor to the SRR, a series of double-ridged waveguides was designed, as shown in Figure 2. The substrate with two SRRs and tuning screws was placed at the center of the filter.

The dimensions of each waveguide section are summarized in Figure 3. The double-ridged rectangular waveguide, with the WRD-200 specifications shown in Figure 3(a), was connected to the adaptor. A quarter-wavelength transformer, as shown in Figure 3(b), was used for impedance matching between the double-ridged rectangular waveguide shown in Figure 3(a) and the double-ridged square waveguide shown in Figure 3(c). The hollow square waveguide shown in Figures 1 and $3(d)$ was connected to the double-ridged square waveguide.

Figure 4(a) shows the equivalent circuit for the onepole waveguide filter loaded with an SRR described in 


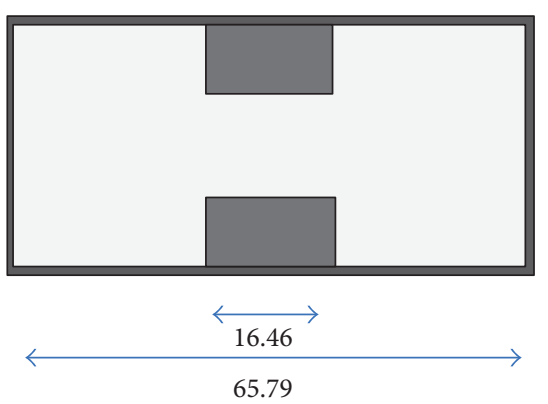

(a)

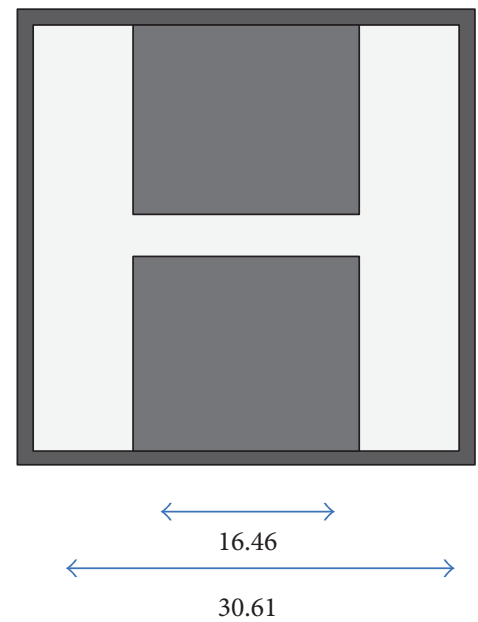

(c)

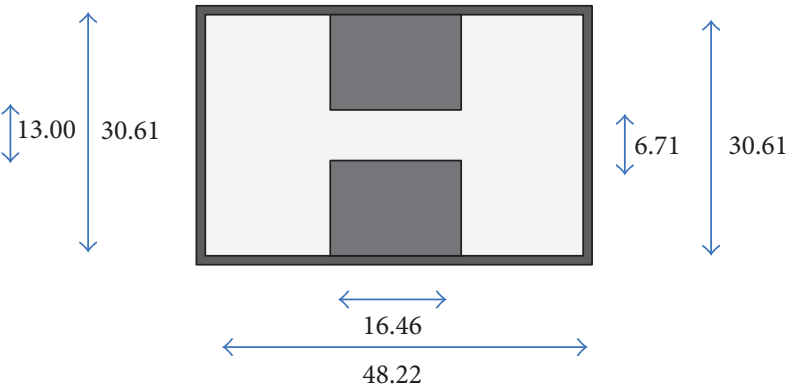

(b)
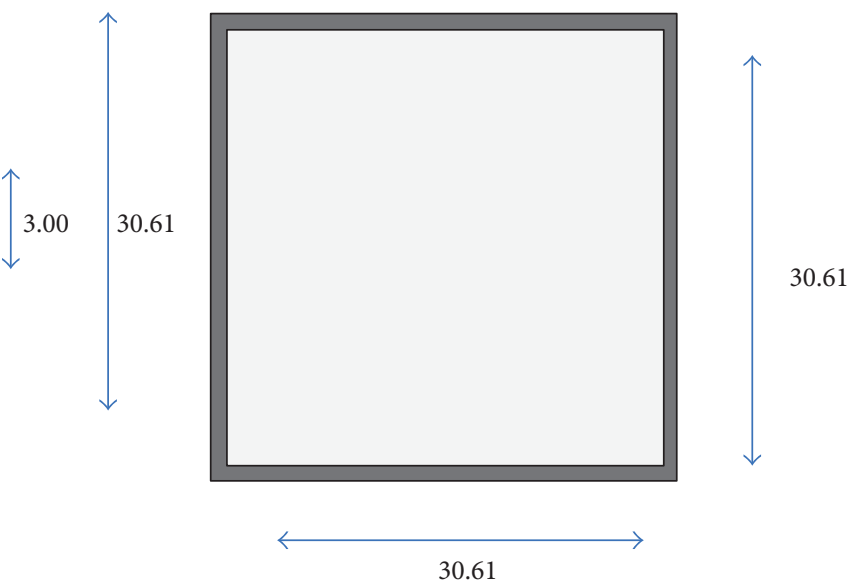

(d)

FIGURE 3: Dimension of each waveguide (unit: mm): (a) WRD-200, (b) transformer, (c) ridge WR, and (d) WR.

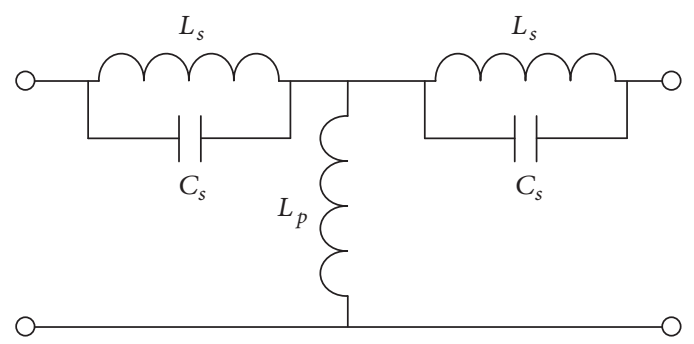

(a)

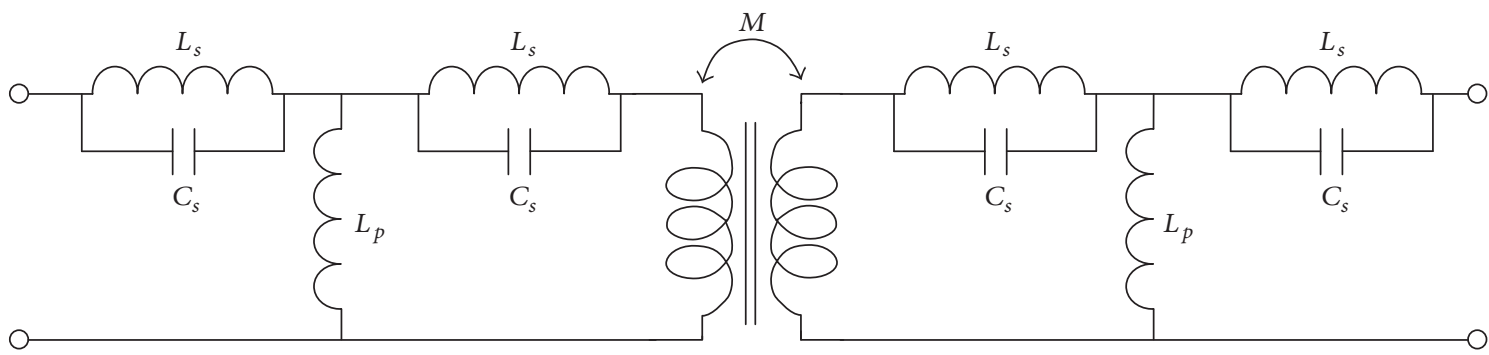

(b)

FIGURE 4: Equivalent circuit of (a) one-pole filter loading a SRR and (b) proposed two-pole filter loading two SRRs. 


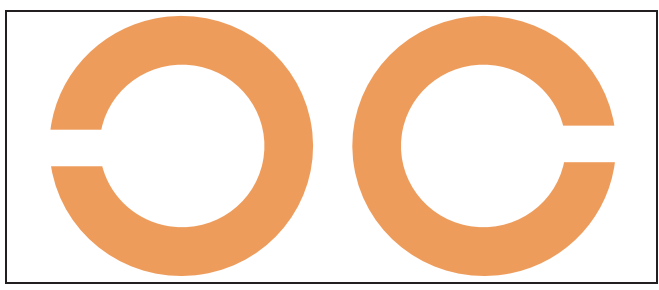

(a)

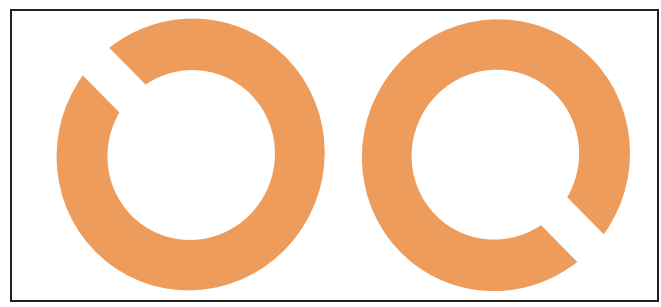

(c)

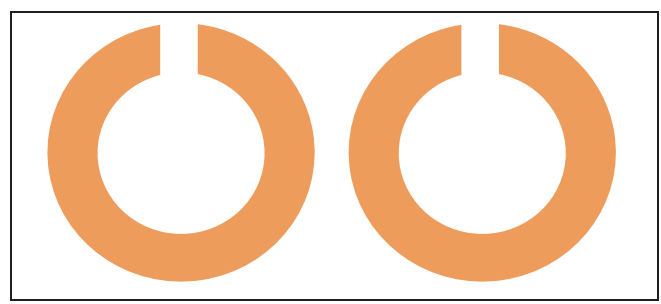

(e)

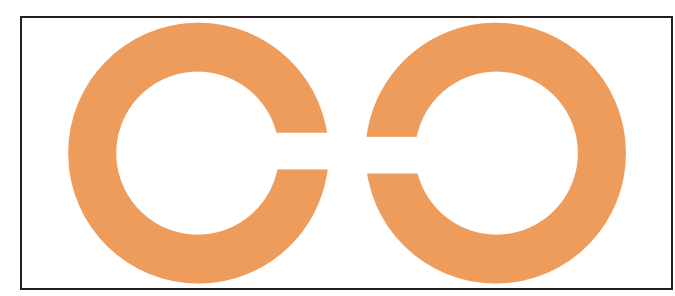

(b)

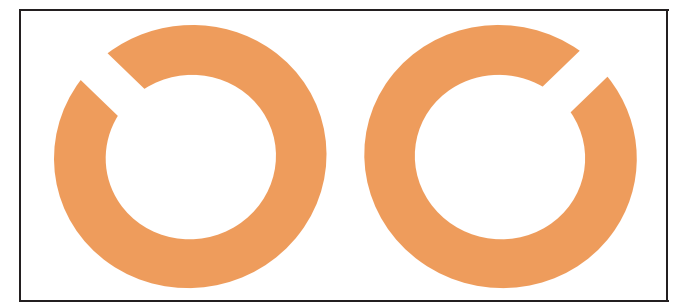

(d)

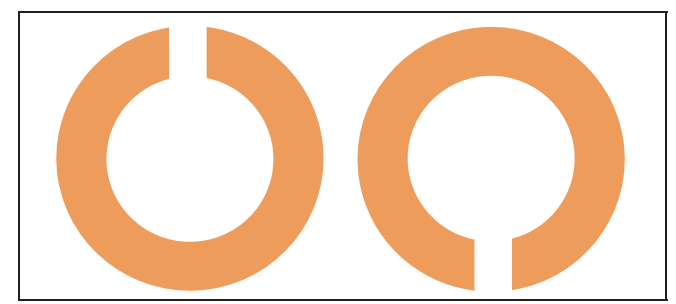

(f)

Figure 5: Different orientation of two rings: (a) $180^{\circ}$ and $0^{\circ}$, (b) $0^{\circ}$ and $180^{\circ}$, (c) $135^{\circ}$ and $-45^{\circ}$, (d) $135^{\circ}$ and $45^{\circ}$, (e) $90^{\circ}$ and $90^{\circ}$, and (f) $90^{\circ}$ and $-90^{\circ}$.

[10], which is composed of a series inductor $\left(L_{s}\right)$, a series capacitor $\left(C_{s}\right)$, and a parallel inductor $\left(L_{p}\right)$. Here, $L_{s}$ and $L_{p}$ are basic components due to the miniaturized waveguide configuration [10]. $C_{s}$ results from the SRR and allows the wave to propagate through the waveguide. The expanded two-pole waveguide filter has the equivalent circuit shown in Figure 4(b). A transformer marked in $M$ was added between the two SRRs [12].

\section{Parametric Analysis and Results}

The proposed waveguide filter was analyzed using commercial software CST Microwave Studio. A range of SSR gaps were investigated, as shown in Figure 5. To classify the ring orientation, the gap position is given in terms of the angle from the $z$-axis, such as $-90^{\circ},-45^{\circ}, 0^{\circ}, 45^{\circ}, 90^{\circ}, 135^{\circ}$, and $180^{\circ}$. For example, Figure 5(a) shows the combination of ring orientations of $180^{\circ}$ and $0^{\circ}$. Others are noted in the caption of Figure 5.

The transmission coefficients $\left(S_{21}\right)$ from the simulation results are shown in Figure 6. As expected from [10], the optimum combination is the structure shown in Figure 5(f). The structures in Figures 5(b)-5(e) show large insertion losses. In contrast, the structure shown in Figure 5(a) has a small insertion loss at $2.1 \mathrm{GHz}$, and sharp skirt characteristics on the right of the $S_{21}$ curve.

The good bandpass characteristics shown in Figure 6 are consistent with the result of the investigation reported in

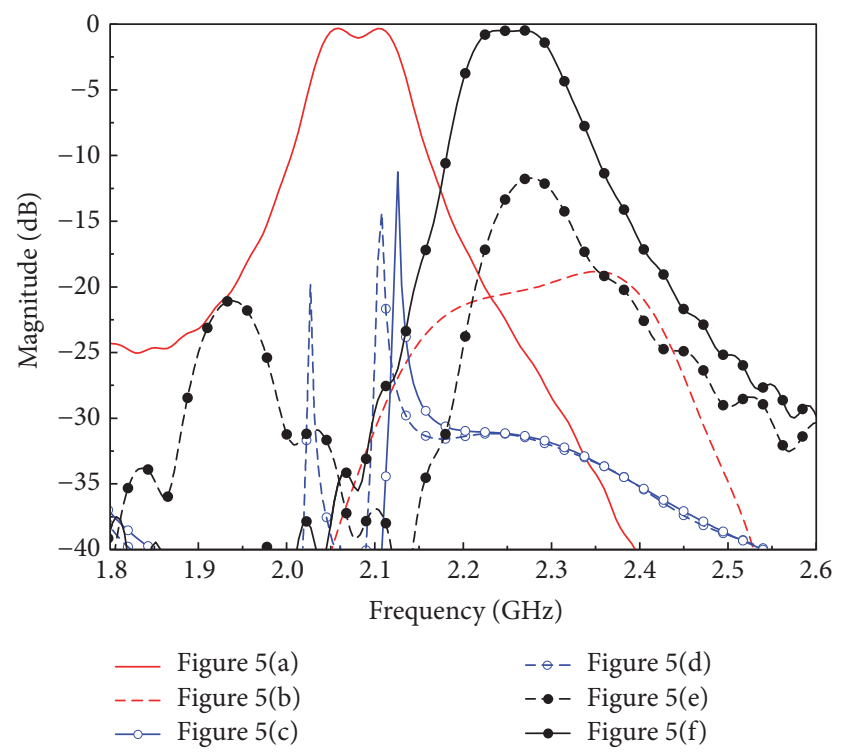

FIGURE 6: Simulated transmission coefficient (S11) with respect to the different orientation of two rings.

[10]. They can be explained from the current distribution at $2.5 \mathrm{GHz}$ shown in Figure 7 . SRRs with a gap positioned upside, as shown in Figure 7(f), have a symmetric current distribution; this allows power delivery from the input to 

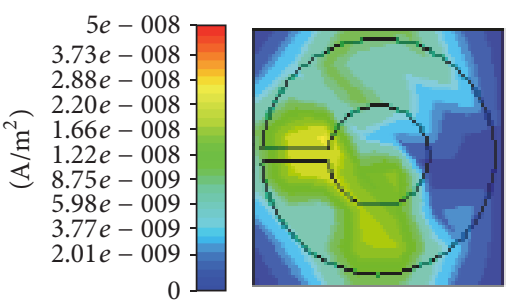

(a)
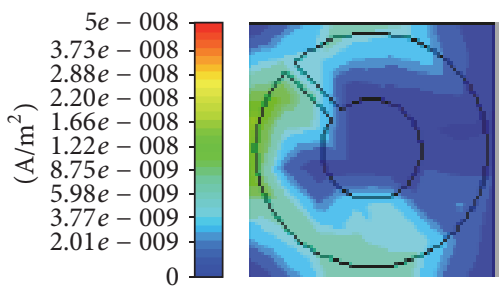

(c)
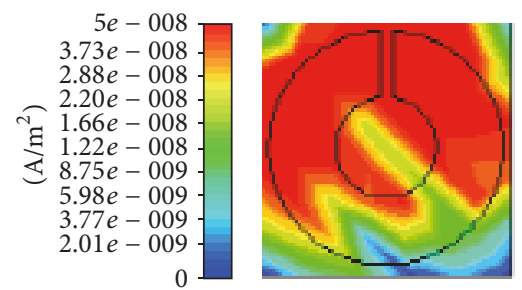

(e)
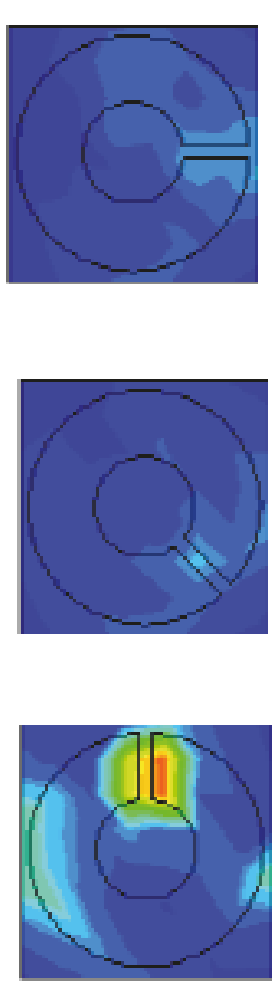

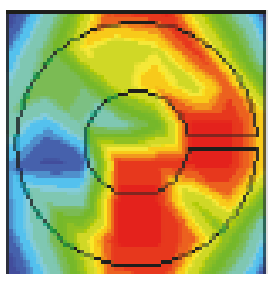

(b)
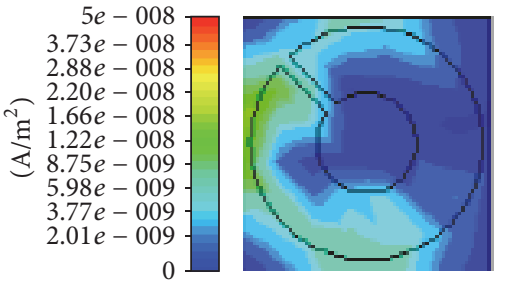

(d)
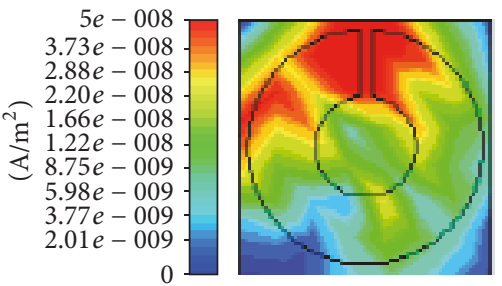

(f)
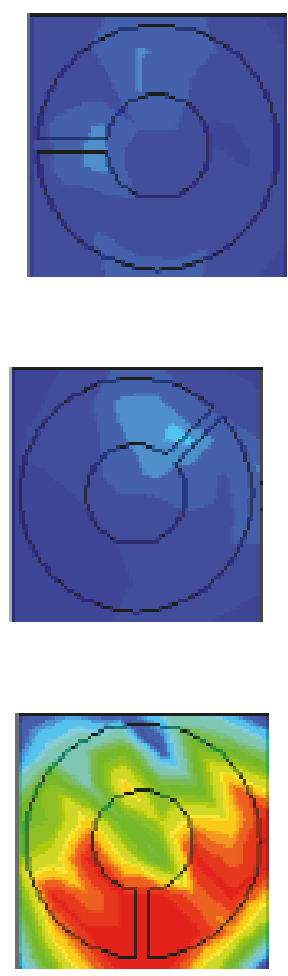

Figure 7: Different orientation of two rings: (a) $180^{\circ}$ and $0^{\circ}$, (b) $0^{\circ}$ and $180^{\circ}$, (c) $135^{\circ}$ and $-45^{\circ}$, (d) $135^{\circ}$ and $45^{\circ}$, (e) $90^{\circ}$ and $90^{\circ}$, and (f) $90^{\circ}$ and $-90^{\circ}$.

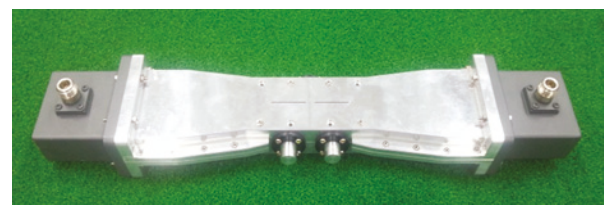

Figure 8: Photograph of the fabricated waveguide filter and measurement setup.

the output port without loss. The other configurations shown in Figures 7(a)-7(e) have asymmetric current distributions, inhibiting waveguide coupling.

A photograph of the fabricated two-pole filter is shown in Figure 8. The central part is the hollow square waveguide showing the tuning screws. The waveguide-to-coaxial adaptors are connected to the waveguides on both sides.

Figures 9(a) and 9(b) show the $S_{11}$ and $S_{21}$ curves obtained by adjusting the spacing between the SRR and the metal screw. The simulated and measured results were compared and showed good agreement for both reflection and transmission. Small differences were attributed to tuning screw misalignment. As the metal screw moves inside with a parameter $s$ value of $4 \mathrm{~mm}$ to $1 \mathrm{~mm}$, the frequency moves upwards from 2.0 to $2.35 \mathrm{GHz}$. The reason is that the magnetic flux is obstructed by the metal screw, which results in a decrease in the effective permeability $[10,11]$. The twopole characteristics were maintained over all frequencies. However, the fractional $3 \mathrm{~dB}$ bandwidth narrowed at higher frequencies. Furthermore, the insertion loss was low in the lower frequency band but slightly higher in the higher frequency band. This is because the quarter-wavelength transformer was optimized for the lower frequency band [10].

\section{Conclusion}

In this paper, frequency tunability with two-pole bandpass characteristics was investigated. The proposed filter was operated below the cutoff frequency, which was possible by use of SRRs. The $S_{21}$ and current distribution characteristics were investigated for a range of SRR gaps and the optimum combination proposed. The equivalent circuit of the proposed two-pole bandpass filter was also investigated alongside that of a one-pole bandpass filter. Simulation and experimental results showed that the frequency could be tuned from 2.0 to $2.35 \mathrm{GHz}$ using tuning screws. As the proposed filter has the advantages of frequency tunability with two-pole bandpass characteristics below the cutoff frequency, it could be applied 


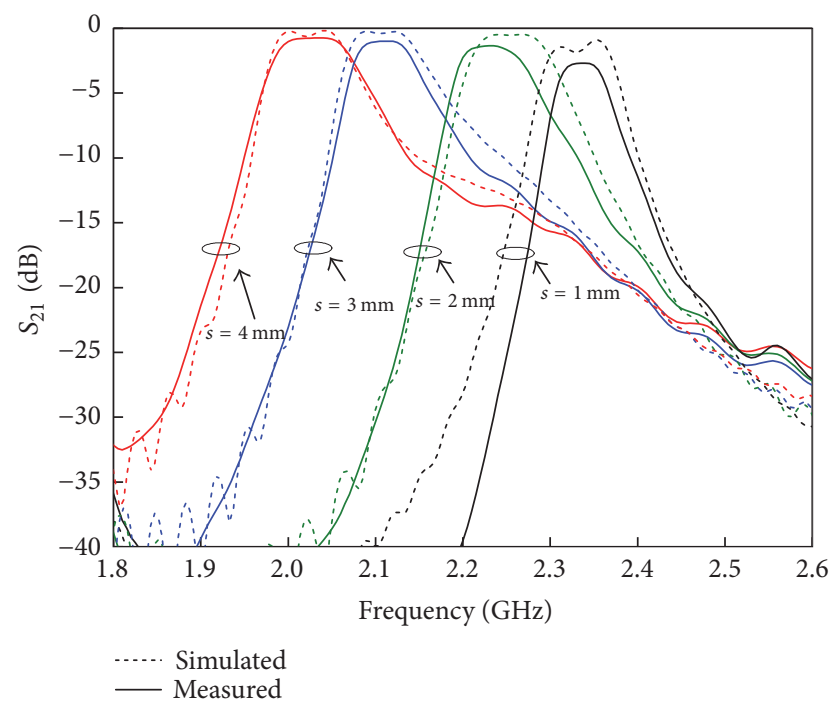

(a)

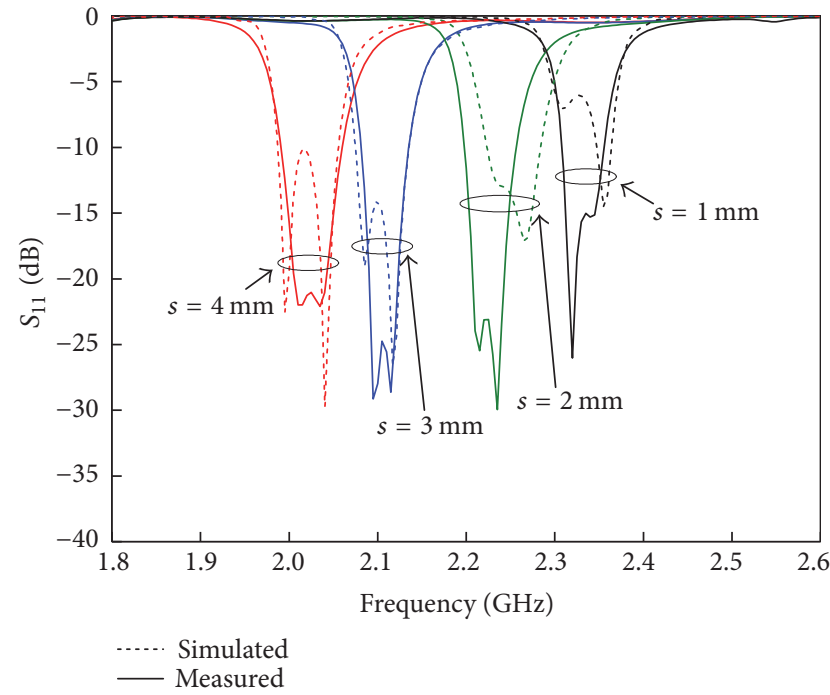

(b)

FIGURE 9: Simulated and measured results for adjusting the gap between SRR and end of tuning screw: (a) reflection coefficient (S11) and transmission coefficient.

to multiband communication systems, such as a softwaredefined radio (SDR) system.

\section{Conflicts of Interest}

The authors declare that they have no conflicts of interest.

\section{Acknowledgments}

This research was supported by Basic Science Research Program through the National Research Foundation of Korea (NRF) funded by the Ministry of Education (no. 2015R1D1A1A01059175) and by research fund from Chosun University in Korea, 2016.

\section{References}

[1] T. Ding, S. Zhang, L. Zhang, and Y. Liu, "Smart cylindrical dome antenna based on active frequency selective surface," International Journal of Antennas and Propagation, vol. 2017, Article ID 5901625, 14 pages, 2017.

[2] A. H. Ramadan, J. Costantine, Y. Tawk, C. G. Christodoulou, and K. Y. Kabalan, "Frequency-tunable and pattern diversity antennas for cognitive radio applications," International Journal of Antennas and Propagation, vol. 2014, Article ID 638627, 7 pages, 2014.

[3] T. K. Wu, "Sharp transition frequency selective surface with concentric double fractal elements," Microwave and Optical Technology Letters, vol. 58, no. 6, pp. 1388-1390, 2016.

[4] J. Beneat and P. Jarry, Advanced Design Techniques and Realizations of Microwave and RF Filters, John Wiley \& Sons, Hoboken, NJ, USA, 2008.

[5] G. Craven, "Waveguide Below Cutoff: a new type of microwave integrated circuit," Microwave Journal, vol. 13, no. 8, p. 51, 1970.

[6] G. F. Craven and C. K. Mok, "The design of evanescent mode waveguide bandpass filters for a prescribed insertion loss characteristic," IEEE Transactions on Microwave Theory and Techniques, vol. 19, no. 3, pp. 295-308, 1971.

[7] M. N. M. Kehn, O. Quevedo-Teruel, and E. Rajo-Iglesias, "Splitring resonator loaded waveguides with multiple stopbands," Electronics Letters, vol. 44, no. 12, pp. 714-716, 2008.

[8] S. Hrabar, J. Bartolic, and Z. Sipus, "Waveguide miniaturization using uniaxial negative permeability metamaterial," IEEE Transactions on Antennas and Propagation, vol. 53, no. 1 I, pp. 110-119, 2005.

[9] G. Lubkowski, C. Damm, B. Bandlow, R. Schuhmann, M. Schußler, and T. Weiland, "Broadband transmission below the cutoff frequency of a waveguide loaded with resonant scatterer arrays," IET Microwaves, Antennas and Propagation, vol. 1, no. 1, pp. 165-169, 2007.

[10] W.-K. Park, S.-T. Han, and S.-S. Oh, "Frequency-tunability of a miniaturized waveguide filter loaded with a split-ringresonator," Microwave and Optical Technology Letters, vol. 55, no. 7, pp. 1649-1653, 2013.

[11] W. H. Hayt, Engineering Electromagnetics, McGraw-Hill, 1989.

[12] R. Marqués, F. Martín, and M. Sorolla, Metamaterials with Negative Parameters: Theory, Design and Microwave Applications, John Wiley \& Sons, Hoboken, NJ, USA, 2008. 


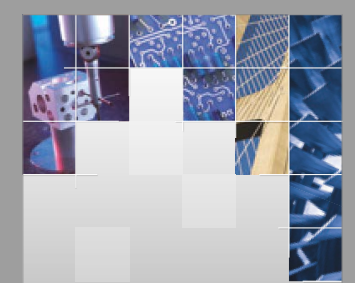

\section{Enfincering}
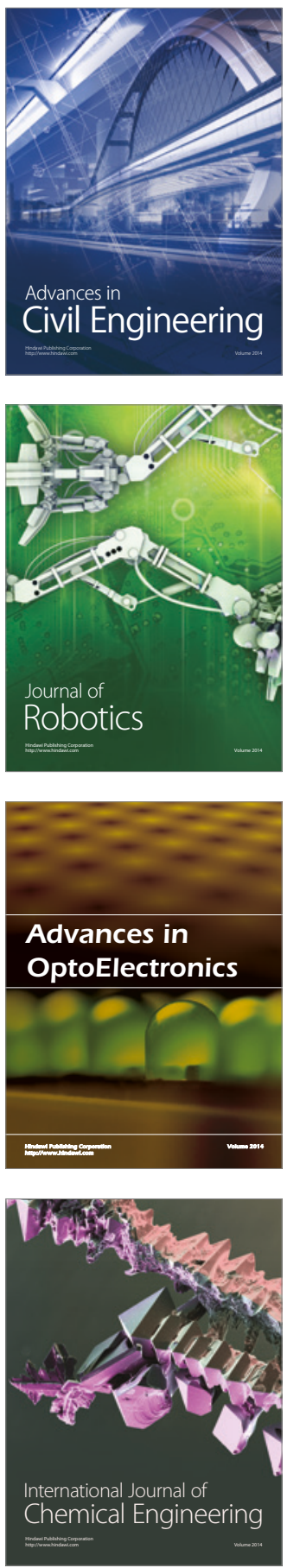

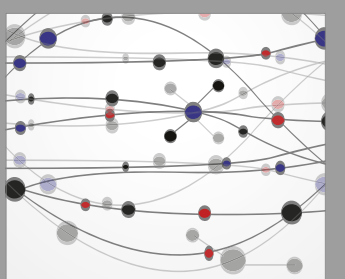

The Scientific World Journal

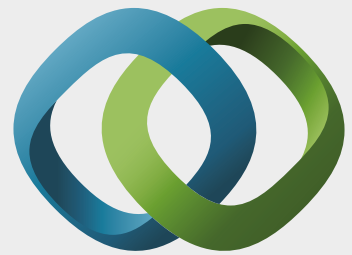

\section{Hindawi}

Submit your manuscripts at

https://www.hindawi.com
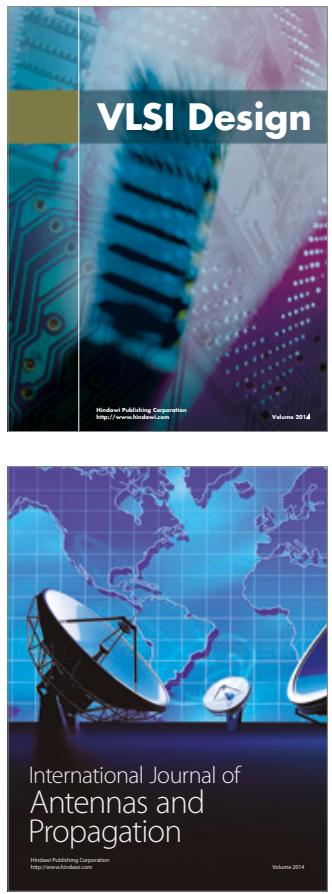

\section{Rotating}

Machinery
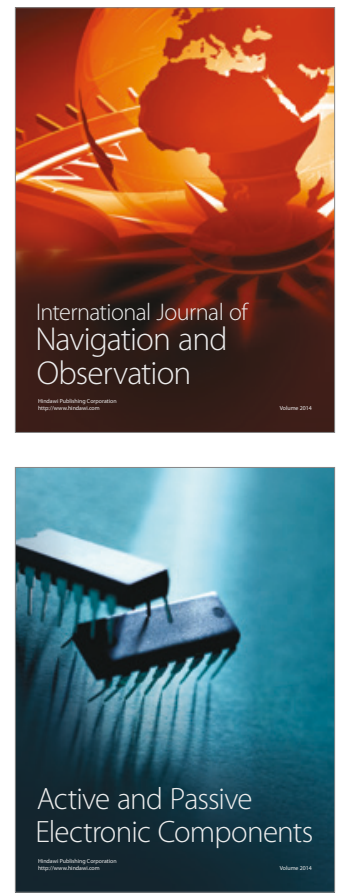
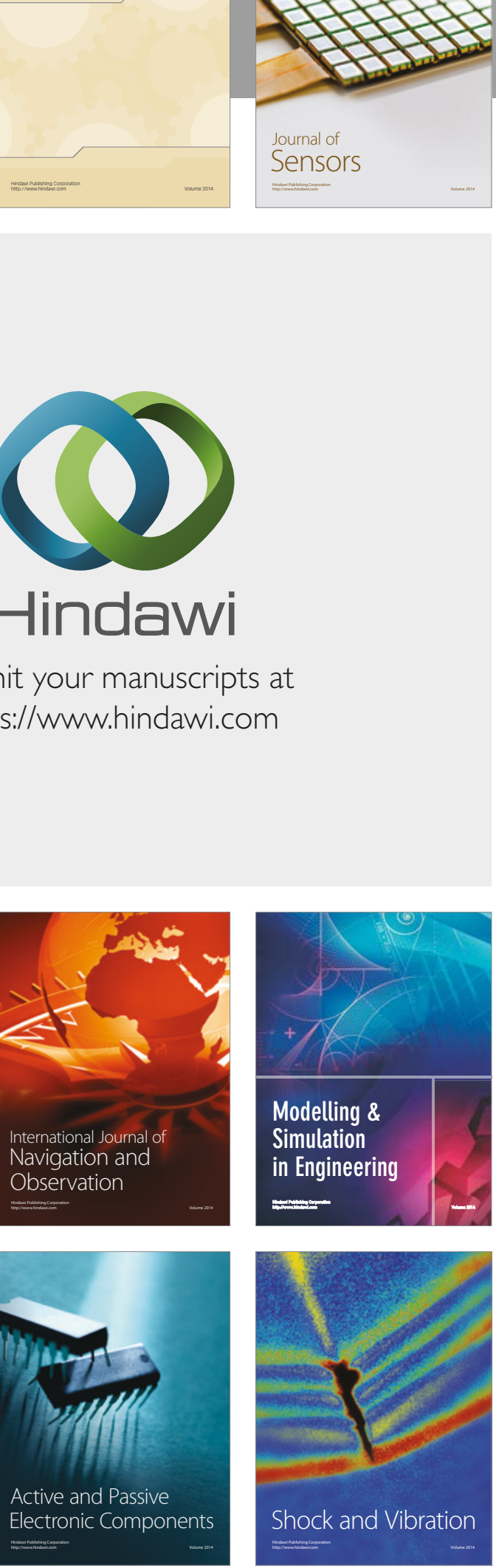
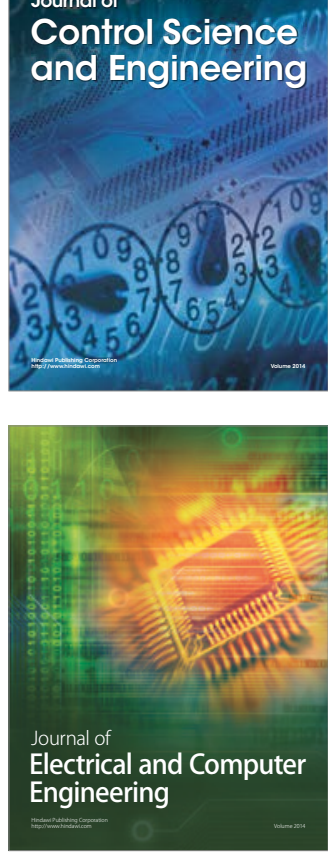

Distributed

Journal of

Control Science

and Engineering
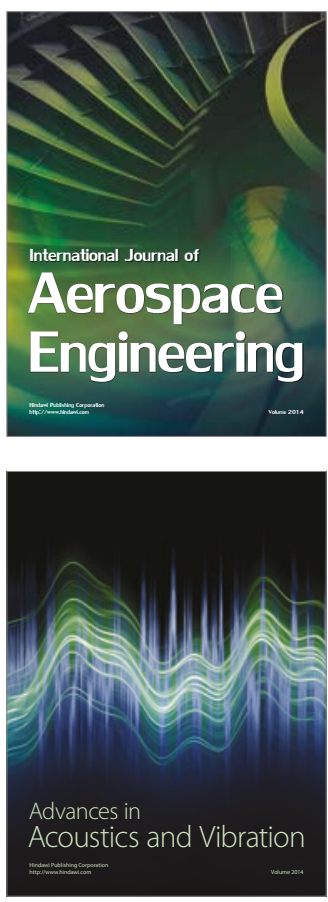

Sensor Networks 